\title{
Entrenamiento Interválico de Alta Intensidad (HIIT) como herramienta terapéutica en pacientes con Diabetes Mellitus Tipo 2: Una revisión narrativa High-Intensity Interval Training (HIIT) as a therapeutic tool in patients with Type 2 Diabetes Mellitus: Anarrative review
}

\author{
Adrián Montes de Oca García, José Gutiérrez Manzanedo, Jesús Gustavo Ponce González
}

Universidad de Cádiz (España)

\begin{abstract}
Resumen. Introducción: El ejercicio físico es imprescindible para las personas con DMT2, aunque, con frecuencia se presentan problemas para practicarlo, siendo el más frecuente la falta de tiempo. No obstante, el auge de métodos como el HIIT aporta una solución a estas barreras, ya que permiten mejorar la salud con unos pocos minutos de ejercicio diarios. Objetivo: Realizar una revisión narrativa sobre los efectos del HIIT en diferentes parámetros de salud en personas con DMT2. Metodología: Tras la búsqueda en bases de datos (PubMed, SPORTDiscus y Medline) se obtiene un total de 52 artículos. Teniendo en cuenta los criterios de inclusión-exclusión, se incluyen 16 artículos. Todos los estudios incluidos son experimentales (ensayos clínicos) publicados en los últimos 10 años. Se excluyen los artículos a los que no se tiene acceso, las revisiones, los estudios cuya intervención sea similar pero no igual al HIIT y aquellos relacionados con genética. Resultados: De total de estudios incluidos (16), hay 14 que afirman que el HIIT mejora la salud de sujetos con DMT2. Se observan mejoras significativas en: perfil lipídico, control glucémico, resistencia a la insulina, HbA1c, tensión arterial, composición corporal, función endotelial y condición física. Hay 2 estudios en los que no se observa ninguna mejora. Conclusiones: El HIIT tiene efectos positivos en la salud de las personas con DMT2, ya que mejora la condición física, la salud cardio-metabólica, la composición corporal y la calidad de vida. El HIIT puede representar una buena estrategia para mejorar la salud en DMT2. Son necesarios más estudios.
\end{abstract}

Palabras clave: Diabetes mellitus tipo 2 (DMT2), Entrenamiento Interválico de Alta Intensidad (HIIT), Resistencia a la insulina y Intolerancia a la glucosa

Abstract. Introduction: Physical exercise is essential for people with T2DM, although there are often problems to practice it, the most frequent being the lack of time. However, the rise of methods such as HIIT provides a solution to these barriers, since they allow improving health with a few minutes of exercise per day. Objective: To carry out a narrative review about the effects of HIIT on different health parameters in people with T2DM. Methodology: After the search in databases (PubMed, SPORTDiscus and Medline) 52 articles were obtained. Taking into account the inclusion-exclusion criteria, 16 articles were included. All included studies are experimental (clinical trials) published in the last 10 years. Excluded are articles that cannot be accessed, reviews, studies whose intervention is similar but not equal to HIIT and those related to genetics. Results: Of the total number of included studies (16), there are 14 that affirm that HIIT improves the health of subjects with T2DM. Significant improvements were observed in: lipid profile, glycemic control, insulin resistance, HbA1c, blood pressure, body composition, endothelial function and physical fitness. There are 2 studies in which no improvement is observed. Conclusions: HIT has positive effects on the health of people with T2DM, since it improves physical fitness, cardio-metabolic health, body composition and quality of life. HIIT may represent a good strategy to improve health in DMT2. More studies are nedeed.

Key words: Type 2 Diabetes Mellitus (T2DM), High-Intensity Interval Training (HIIT), Insulin Resistance, Impaired glucose tolerance.

\section{Introducción}

La diabetes mellitus es una patología crónica caracterizada por desórdenes en el funcionamiento normal del metabolismo de la glucosa, aunque también existen anomalías en el metabolismo lipídico y proteico (López Chicharro \& Mojares, 2008). Se trata de una afección que aparece cuando las células $\beta$ del páncreas no producen suficiente insulina o cuando el organismo no consigue captar la glucosa a nivel celular debido a una situación de insulinorresistencia. Por tanto, un fallo en la producción de insulina, en la acción de esta o en ambas cosas, conllevará un aumento de los niveles de glucosa en el torrente sanguíneo (hiperglucemia) (López Chicharro \& Mojares, 2008).

La diabetes mellitus tipo 2 (DMT2) es la forma más común de diabetes, aunque también existen la diabetes mellitus tipo 1 (enfermedad autoinmune caracterizada por la destrucción de las células $\beta$, productoras de insulina en el páncreas,

Fecha recepción: 15-01-19. Fecha de aceptación: 03-05-19 Jesús Gustavo Ponce González

jesusgustavo.ponce@uca.es sintetizándose muy poca o ninguna insulina), la diabetes mellitus gestacional y otros tipos de diabetes mellitus debidos principalmente a factores genéticos. La DMT2 es un tipo de diabetes no dependiente de insulina, ya que el páncreas produce insulina correctamente, pero cuando ésta se une a su receptor en la célula para hacer la captación de glucosa a través de la proteína GLUT4, la cascada de señalización está alterada, impidiendo la función normal de la hormona. Debido a la defectuosa captación de glucosa, el páncreas detecta que sigue habiendo un aumento de glucemia en sangre, liberando más cantidad de insulina (hiperinsulinemia). Este exceso de trabajo por parte del páncreas se relaciona en el tiempo con un déficit parcial o completo en los peores casos en la producción de insulina, provocando que se conviertan en insulinodependientes (López Chicharro \& Mojares, 2008). La DMT2 constituye el 85\% de la población con diabetes y es más común en hombres. Además, está íntimamente relacionada con el sobrepeso y la obesidad. De hecho, una de las hipótesis más aceptadas es que el acúmulo de triglicéridos en el interior de las células produce la ruptura de la cascada de señalización de la hormona insulina debido a la lipotoxicidad. En general, los sín- 
tomas clave de la DMT2 son: resistencia a la insulina (medida utilizando el HOMA-IR, Homeostasis Model Assessment for Insulin Resistance por sus siglas en inglés) (Geloneze, Repetto, Geloneze, Tambascia, \& Ermetice, 2006) hiperinsulinemia, hiperglucemia, poliuria, polidipsia, polifagia y pérdida de peso inexplicable (Inzucchi \& Sherwin, 2012).

Los criterios de diagnóstico de la DMT2 aparecen detalladamente a continuación (López Chicharro \& Mojares, 2008):

1. Glucemia al azar $\geq 200 \mathrm{mg} / \mathrm{dl}$ en presencia de síntomas de diabetes.

2. Glucemia en ayunas (al menos durante 8 horas) $\geq 126$ $\mathrm{mg} / \mathrm{dl}$.

3. Glucemia $\geq 200 \mathrm{mg} / \mathrm{dl}$ a las 2 horas tras la prueba de tolerancia oral a la glucosa (OGTT, por sus siglas en inglés) con 75 gramos.

4. Hemoglobina glucosilada $(\mathrm{HbA} 1 \mathrm{c}) \geq 6.5 \%$ (Kumar et al., 2010).

No obstante, hay que considerar también el estado de prediabetes o intolerancia a la glucosa, en el cual el sujeto no llega a presentar síntomas de enfermedad, aunque sí valores superiores a los normales:

1. Glucemia en ayunas (al menos durante 8 horas) $\geq 100$ $\mathrm{mg} / \mathrm{dl} \mathrm{y}<126 \mathrm{mg} / \mathrm{dl}$.

2. Glucemia $\geq 140 \mathrm{mg} / \mathrm{dl} \mathrm{y}<200 \mathrm{mg} / \mathrm{dl}$ a las 2 horas tras la prueba de tolerancia oral a la glucosa (OGTT, por sus siglas en inglés) con 75 gramos.

3. Hemoglobina glucosilada (HbA1c) 5.7-6.4\% (Kumar et al., 2010).

En lo referente al tratamiento de la patología, puede tratarse farmacológicamente con antidiabéticos orales dependiendo de la gravedad, aunque la dieta y el ejercicio son las bases fundamentales de éste. La dieta debe ser individualizada y equilibrada, y debe priorizar el consumo de carbohidratos complejos de alta carga glucémica y la ingesta de alimentos ricos en fibra, proteínas y ácidos grasos poliinsaturados y, sobre todo, monoinsaturados (ácido oleico: aceite de oliva virgen), así como reducir el consumo de alcohol, sodio y, sobre todo, azúcares simples y alimentos ricos en grasas saturadas (Evert et al., 2013). De hecho, recientes estudios han seleccionado la dieta mediterránea como una de las mejores estrategias nutricionales actuales para prevenir y tratar la diabetes mellitus (Esposito et al., 2015; Salas-Salvadó et al., 2014).

Con respecto al ejercicio, en los últimos años se han publicado numerosos estudios científicos que demuestran los grandes beneficios del ejercicio físico sobre la salud cardiovascular y metabólica y la calidad de vida de los sujetos con DMT2 y prediabetes, sobre todo si éste se combina con dieta reglada y si el tiempo de práctica a la semana es superior a 150 minutos (Umpierre, 2011). Teniendo en cuenta el tipo de ejercicio físico, tanto el ejercicio aeróbico como el de fuerza muscular conllevan un gran número de adaptaciones orgánicas agudas y crónicas que ocurren a nivel cromosómico, metabólico y neuromuscular, jugando el músculo esquelético un papel esencial en la mediación de dichas adaptaciones. De hecho, lo más adecuado en pacientes con diabetes es realizar un entrenamiento combinado, llevando un control glucémico continuo (Baños, 2016). Sin embargo, existen diferencias entre ambas modalidades de ejercicio.
El ejercicio aeróbico se relaciona con el aumento del $\mathrm{VO}_{2}$ max, la capacidad oxidativa y la sensibilidad a la insulina (posiblemente debido a la mayor oxidación de triglicéridos en el interior de la célula, entre otras causas gracias a un aumento de la actividad de AMPK o proteína quinasa activada por AMP y al aumento de la biogénesis mitocondrial) y la reducción de la HbA1c, la masa grasa y el riesgo cardiovascular. Por otro lado, el ejercicio de fuerza muscular (ejercicios contra resistencia) se relaciona con la mejora de la tolerancia a la glucosa y la sensibilidad a la insulina (debido a una normalización de la cascada de señalización y la translocación del GLUT4 a la membrana plasmática celular, junto con un incremento de las proteínas de la cascada de señalización, como el GLUT4 intracelular, y el aumento de la fosforilación de proteínas fundamentales en la cascada como AS160), el aumento de la fuerza y la masa magra (debido a un aumento en la síntesis de proteínas), la reducción de la masa grasa y el aumento del metabolismo basal (entre otras causas debido a un aumento de la actividad de AMPK) (Zanuso et al., 2017).

Por consiguiente, el ejercicio de fuerza y potencia muscular es una estrategia muy efectiva de tratamiento de la neuropatía periférica diabética (daño de los nervios periféricos que afecta principalmente a las piernas y a los pies), ya que reduce la pérdida de axones motores y frena los cambios estructurales de las fibras musculares. No obstante, cuando se combinan el ejercicio aeróbico y el ejercicio de fuerza los beneficios en cuanto al control glucémico son mayores que cuando se trabajan la fuerza y la capacidad aeróbica de forma aislada (Baños, 2016; Zanuso et al., 2017).

Por lo tanto, se hace evidente que el ejercicio combinado debe ser una parte fundamental de la vida de las personas con DMT2 y prediabetes y también de las personas sanas, ya que la inactividad física y el sedentarismo están relacionados con un aumento del riesgo de padecer DMT2 o prediabetes, por lo que la actividad física y el ejercicio juegan un papel crucial en la prevención de éstas (Baños, 2016; Zanuso et al., 2017). También podrían ser efectivas otras estrategias como el ejercicio acuático, ya que ha demostrado ser efectivo para tratar el síndrome metabólico, sobre todo en lo relativo a la reducción de los triglicéridos (OchoaMartínez et al., 2019).

Sin embargo, con frecuencia se presentan problemas o barreras para practicar ejercicio, siendo el más común la falta de tiempo debido a la carga familiar y laboral. Estos problemas se acentúan más en sujetos con DMT2 ya que en muchas ocasiones sienten rechazo hacia la práctica de ejercicio por miedo a sufrir accidentes como hipoglucemias, mareos o hipotensión inducida por el ejercicio físico. No obstante, el actual auge de eficientes métodos de entrenamiento como el Entrenamiento Interválico de Alta Intensidad (HIIT) aporta una gran solución, ya que éstos brindan la posibilidad de mejorar la salud y la condición física con solo unos pocos minutos diarios de entrenamiento intenso (Buchheit \& Laursen, 2013).

El HIIT puede realizarse tanto en tapiz rodante como en cicloergómetro, aunque el cicloergómetro ofrece la ventaja de evitar los impactos osteoarticulares y, por tanto, puede ser más recomendable para personas obesas. Asimismo, también puede realizarse en otros aparatos de entrenamiento 
como la elíptica. En lo referente a las numerosas variables que definen y determinan las adaptaciones, cabe destacar que el HIIT se basa en un entrenamiento de bajo volumen compuesto por intervalos de alta intensidad e intercalado con intervalos de recuperación de media o baja intensidad. El tiempo de trabajo, el número de intervalos, la intensidad y el tiempo de descanso variarán en función del objetivo y del nivel de condición física del participante (Buchheit \& Laursen, 2013). Por lo general, cuanto mayor sea la intensidad de los intervalos y menor sea el nivel de condición física, mayor será el tiempo de recuperación.

Por tanto, el principal objetivo es realizar una revisión narrativa sobre los efectos del HIIT en diferentes parámetros de salud en personas con DMT2: condición física, salud cardiovascular y metabólica (control glucémico, HbA1c, resistencia a la insulina, perfil lipídico, tensión arterial y función endotelial), composición corporal y calidad de vida.

\section{Metodología}

Las bases de datos utilizadas fueron PubMed, SPORTDiscus y Medline, introduciendo los términos MeSH y el operador booleano (AND): «High-Intensity Interval Training» AND «Type 2 Diabetes Mellitus». A la búsqueda realizada se le aplica el filtro de los últimos 10 años, para centrarnos en las últimas actualizaciones.

- PubMed (MeSH): 12 artículos.

- SPORTDiscus: 5 artículos.

- Medline: 35 artículos.

Finalmente, se obtiene tras la búsqueda un total de 52 artículos científicos de todas las bases de datos utilizadas.

Criterios de inclusión

- Debían ser estudios experimentales (ensayos clínicos).

- Estudios publicados en los últimos 10 años (2008-2018).

Aplicando estos criterios se obtiene un total de 20 artículos científicos.

Criterios de exclusión

- Accesibilidad de los artículos (artículos de pago): 2 artículos.

- Estudios de revisión (narrativa o sistemática): 1 artículo.

- Intervenciones similares pero no iguales al HIIT, como el HIIT de esfuerzo reducido (REHIIT, por sus siglas en inglés): 1 artículo.

- Estudios relacionados con genética: 1 artículo.

En total se excluyen 5 artículos, por lo que el resultado

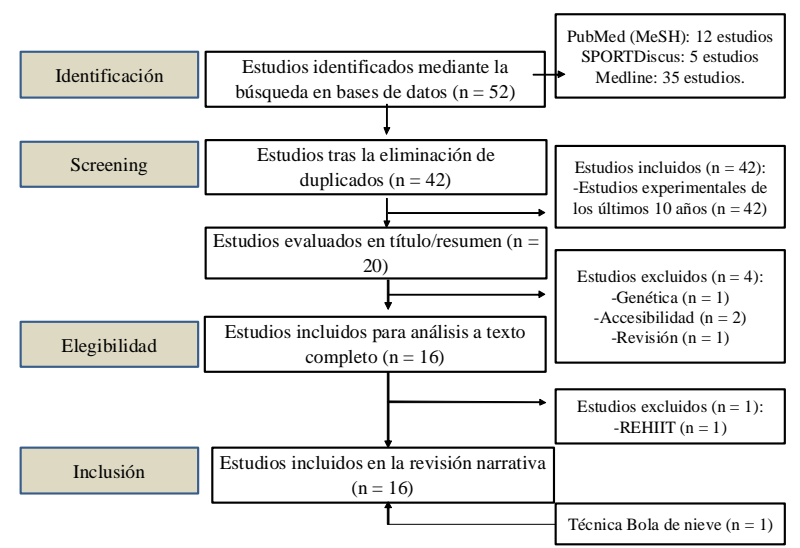

final es de 15 estudios.

Finalmente, se aplica la técnica de la «Bola de nieve» y se añade 1 artículo más a la revisión tras la lectura de las referencias. Por lo tanto, el número definitivo de estudios incluidos en la revisión es de 16 artículos (Figura 1).

\section{Resultados}

En la Tabla 1 se exponen detalladamente las características de los estudios incluidos en la revisión (16). De total de estudios incluidos en esta revisión narrativa, hay 14 (Alvarez et al., 2016; Fex, Leduc-Gaudet, Filion, Karelis, \& AubertinLeheudre, 2015; Francois, Durrer, Pistawka, Halperin, \& Little, 2016; Gillen et al., 2012; Honkala et al., 2017; Little et al., 2011; Madsen et al., 2015b; Madsen et al., 2015c; Maillard et al., 2016; Mandrup et al., 2017; Mangiamarchi et al., 2017; Phillips et al., 2017; Poblete Aro, Russell Guzmán, Soto Muñoz, \& Villegas González, 2015; Støa et al., 2017) que afirman que el HIIT mejora la salud de los sujetos con DMT2 o prediabetes, ya que se observan notables mejoras en: perfil lipídico, control glucémico, insulinorresistencia, HbA1c, tensión arterial y composición corporal. Dentro de estos estudios, encontramos además uno que afirma que el HIIT mejora la calidad de vida (Mangiamarchi et al., 2017) y otro que defiende la efectividad de HIIT en la normalización del estrés oxidativo (Poblete Aro et al., 2015).

Sin embargo, hay 2 estudios en los que no se observan mejoras. Uno de ellos (Aguilera et al., 2015) concluye que el HIIT no mejora significativamente los niveles de HbA1c, aunque sí la composición corporal y la condición física, de forma similar al entrenamiento continuo. El otro estudio (Madsen et al., 2015a) concluye que son necesarias más intervenciones de entrenamiento a largo plazo para disminuir las concentraciones de ácidos grasos libres y, sobre todo, de citoquinas inflamatorias, ya que las mejoras obtenidas son observables, pero no significativas.

Asimismo, cabe destacar que, del total de estudios incluidos, hay uno (Mangiamarchi et al., 2017) que demuestra que los efectos del HIIT son mayores cuando el entrenamiento se combina con dieta (educación nutricional).

Por otro lado, hay 5 estudios (Aguilera et al., 2015; Honkala et al., 2017; Maillard et al., 2016; Poblete Aro et al., 2015; Støa et al., 2017) que comparan el HIIT con el entrenamiento continuo (MICT). De estos 5 estudios, encontramos 4 (Honkala et al., 2017; Maillard et al., 2016; Poblete Aro et al., 2015; Støa et al., 2017) que afirman que los beneficios para la salud son mayores con el HIIT. Sólo un estudio (Aguilera et al., 2015) afirma que no hay diferencias entre el HIIT y el entrenamiento continuo en cuanto a beneficios para la condición física y la composición corporal.

Encontramos también 2 artículos que hablan sobre los efectos del HIIT en mujeres con menopausia y/o postmenopausia (Maillard et al., 2016; Mandrup et al., 2017). El primer artículo (Mandrup et al., 2017) afirma que 3 meses de HIIT reducen los factores de riesgo de DMT2 en premenopáusicas y postmenopáusicas. Por otro lado, el segundo artículo (Maillard et al., 2016) afirma que el HIIT es más efectivo que el entrenamiento continuo para reducir la obesidad central en postmenopáusicas.

Asimismo, un estudio científico de la revisión (Alvarez 


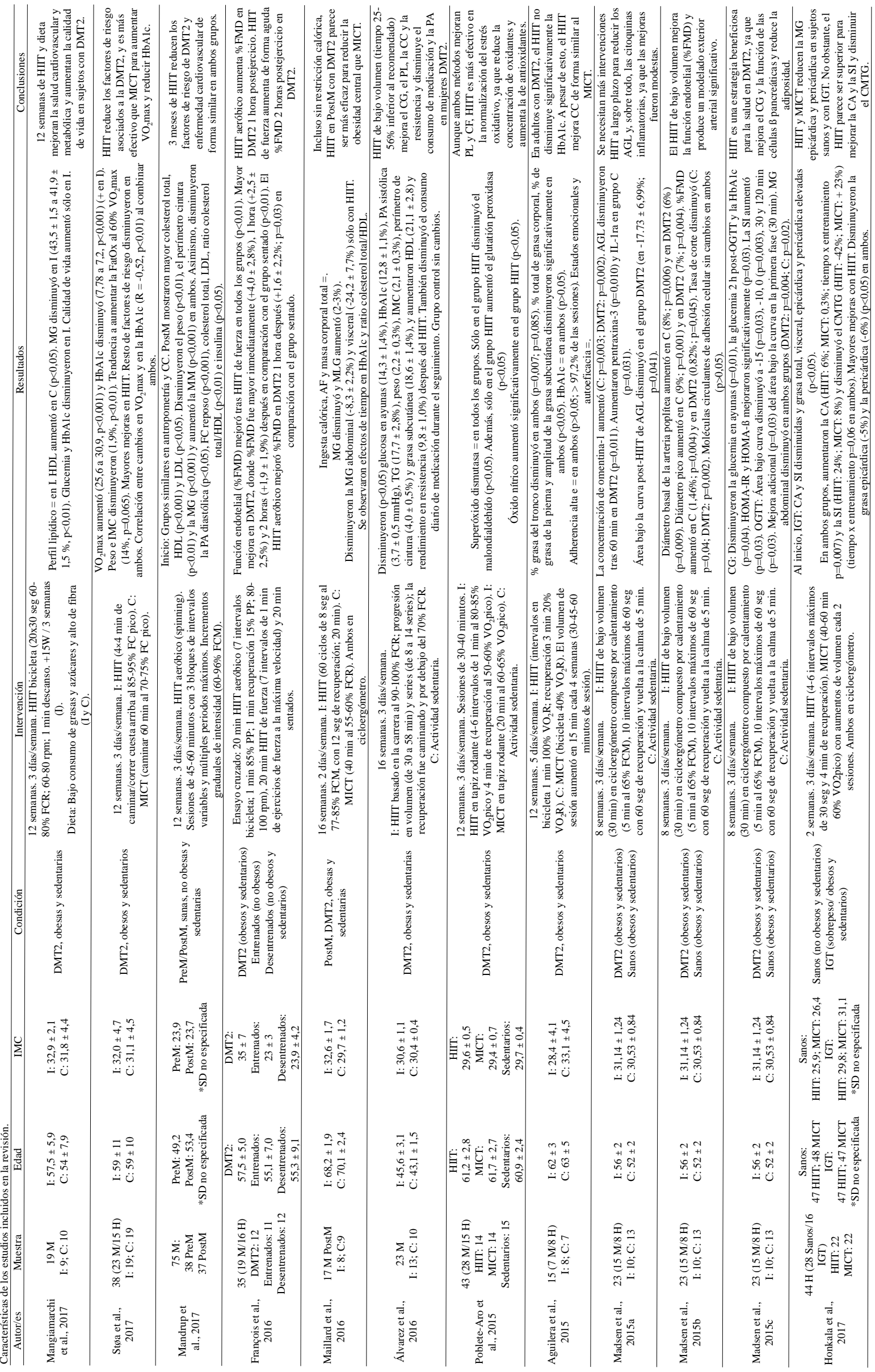




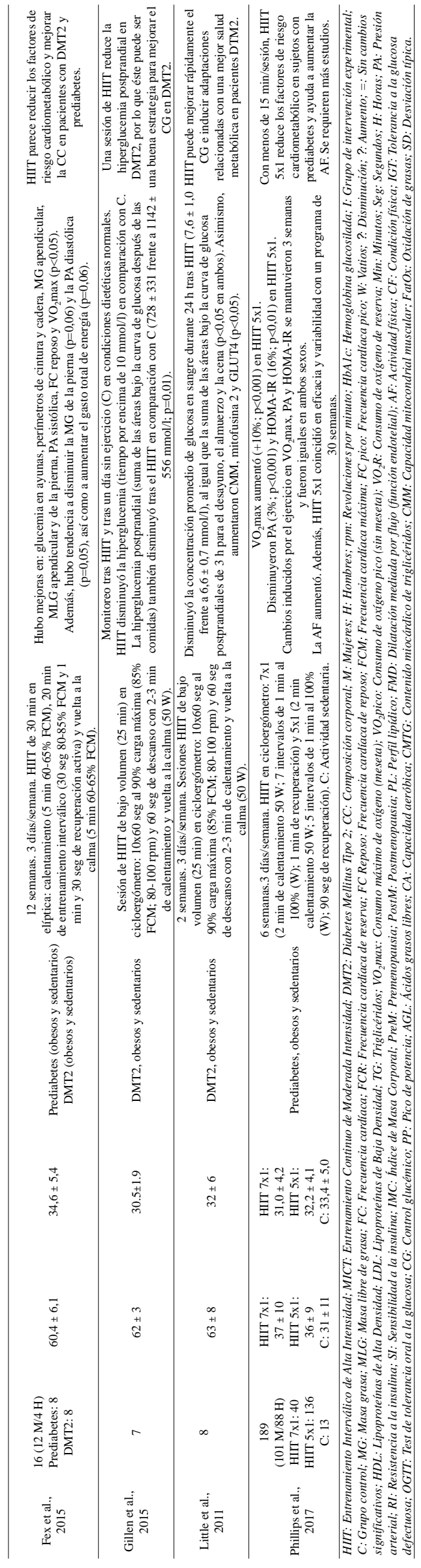

et al., 2016) concluye que el entrenamiento HIIT en personas con DMT2 reduce el consumo de medicamentos en esta población, por lo que se demuestra que el Entrenamiento Interválico de Alta Intensidad puede ser una mejor alternativa de tratamiento que el uso de fármacos antidiabéticos orales.

Observamos también 2 estudios (Francois et al., 2016; Madsen et al., 2015b) de esta revisión que afirman que el HIIT mejora la función endotelial (\% FMD o dilatación mediada por flujo) de las personas con DMT2.

En lo relativo al efecto agudo del entrenamiento, cabe destacar también que hay 2 artículos (Francois et al., 2016; Gillen et al., 2012) que demuestran que el HIIT mejora la función endotelial y reduce la hiperglucemia de forma aguda o inmediata en individuos con DMT2.

Con respecto al HIIT de bajo volumen, encontramos en esta revisión 7 estudios científicos (Alvarez et al., 2016; Gillen et al., 2012; Little et al., 2011; Madsen et al., 2015a; Madsen et al., 2015b; Madsen et al., 2015c; Phillips et al., 2017) con esta metodología. De estos estudios, todos excepto uno (Madsen et al., 2015a) obtienen mejoras significativas en el perfil lipídico, el control glucémico, la insulinorresistencia, la HbA1c, la tensión arterial y la composición corporal de los sujetos con DMT2 o prediabetes.

Finalmente, cabe mencionar que, del total de estudios científicos incluidos en la revisión, sólo un estudio (Francois et al., 2016) incluye ejercicios de fuerza muscular en la metodología HIIT, estando el resto de las metodologías basadas únicamente en ejercicios de tipo aeróbico (cicloergómetro o tapiz rodante). Por lo tanto, esto deberá tenerse en cuenta en futuros estudios científicos que investiguen los efectos del HIIT sobre la DMT2.

\section{Discusión}

A partir de los resultados obtenidos en esta revisión narrativa se puede afirmar que el entrenamiento HIIT mejora la salud de los sujetos con DMT2 o prediabetes (condición física, salud cardio-metabólica, composición corporal y calidad de vida), aunque la evidencia científica es algo limitada, ya que sólo hay 16 artículos en esta revisión, de los cuales 14 (Alvarez et al., 2016; Fex et al., 2015; Francois et al., 2016; Gillen et al., 2012; Honkala et al., 2017; Little et al., 2011; Madsen et al., 2015b; Madsen et al., 2015c; Maillard et al., 2016; Mandrup et al., 2017; Mangiamarchi et al., 2017; Phillips et al., 2017; Poblete Aro et al., 2015; Støa et al., 2017) confirman los beneficios del HIIT sobre la DMT2, mientras que hay 2 estudios (Aguilera et al., 2015; Madsen et al., 2015a) que contradicen los resultados de los anteriores, aunque esto bien puede deberse a un diseño diferente de la metodología de entrenamiento. Por lo tanto, el nivel de evidencia científica no puede ser considerado alto, entre otros motivos porque la metodología HIIT es relativamente reciente y porque hay pocos estudios científicos que investiguen los efectos de este sobre la DMT2, por lo que este aspecto deberá tenerse en cuenta de cara a futuras investigaciones sobre el tema.

Con respecto al diseño de las metodologías de entrenamiento (intervención o tratamiento), cabe destacar que el diseño predominante y el más efectivo es el entrenamiento HIIT de tipo aeróbico en cicloergómetro de 12 semanas (3 
sesiones por semana), con una intensidad en torno al 8590\% FCM/VO pico y 80-100 rpm y un volumen bajo (30 minutos por sesión aproximadamente). Sin embargo, este tipo de diseños metodológicos conlleva una serie de limitaciones que han de tenerse muy en cuenta.

En primer lugar, hay que tener en cuenta que, tratándose de un entrenamiento de alta intensidad con intervalos de corta duración, utilizar la frecuencia cardíaca o el consumo de oxígeno para medir la intensidad del ejercicio no es lo más idóneo, ya que en un intervalo corto de tiempo es difícil alcanzar frecuencias cardíacas cercanas a la máxima, sobre todo tratándose de personas sedentarias y con una condición física baja. De hecho, lo más adecuado y fiable para este tipo de diseños sería utilizar el pico de potencia o carga máxima de trabajo (cuantificado en vatios), tal y como se ha hecho en 4 de los estudios incluidos en esta revisión (Francois et al., 2016; Gillen et al., 2012; Little et al., 2011; Phillips et al., 2017).

En segundo lugar, otra limitación de este tipo de diseño es que no se incluyen en ningún momento ejercicios de fuerza muscular. De hecho, en esta revisión sólo hay un estudio (Francois et al., 2016) cuya metodología contempla esta capacidad física. Por lo tanto, se hace evidente que son necesarios más estudios que tengan en cuenta, dentro de la metodología HIIT, la fuerza muscular y no sólo la capacidad aeróbica en el diseño de sus intervenciones, ya que ambas son igualmente importantes de cara a una mejora de la salud de las personas con DMT2 o prediabetes. A su vez, el trabajo de fuerza muscular previene la pérdida de masa muscular en esta población, quienes tienen sarcopenia debido al catabolismo de las proteínas para conseguir energía al no poder captar la glucosa plasmática.

En lo referente al tiempo de la sesión, cabe destacar que sólo 2 estudios (Aguilera et al., 2015; Mandrup et al., 2017) sobrepasan los 30 minutos de sesión, lo que indica que el HIIT, con menos volumen de entrenamiento, puede ser más beneficioso que otras metodologías con mayor volumen de sesión como el MICT. Por tanto, se hace evidente que el HIIT puede ser una herramienta terapéutica muy beneficiosa y útil para aquellas personas con DMT2 que disponen de poco tiempo al día para hacer ejercicio y que, además, prefieren metodologías de entrenamiento alternativas.

Por otro lado, con respecto a las características de los participantes se observa que la edad de los sujetos en los 16 estudios es de más de 18 años. De hecho, a pesar de que la edad predominante de los sujetos sea de unos 50 años aproximadamente, 8 artículos (Aguilera et al., 2015; Fex et al., 2015; Gillen et al., 2012; Little et al., 2011; Maillard et al., 2016; Phillips et al., 2017; PobleteAro et al., 2015; Støa et al., 2017) incluyen además a sujetos que llegan a sobrepasar los 65 años, es decir, personas mayores. Por lo tanto, esta revisión no sólo ofrece información relativa a personas adultas, sino que también proporciona datos sobre los efectos del HIIT en adultos mayores de 65 años que padecen DMT2 o prediabetes. No obstante, sería recomendable que de cara a futuras intervenciones experimentales con ejercicio físico se tenga más en cuenta la diferencia de edad de los sujetos de la muestra, ya que los resultados pueden ser muy diferentes en una misma muestra de individuos, sobre todo si algunos están en edad adulta (18-64 años) y otros en edad anciana (más de 65 años). Asimismo, también sería interesante investigar los efectos del HIIT en sujetos menores de 18 años que padecen DMT2 o prediabetes, ya que cada vez existen más casos debido al aumento de la prevalencia de obesidad en niños y adolescentes en las últimas décadas (Dehghan, Akhtar-Danesh, \& Merchant, 2005; Ogden, Carroll, Kit, \& Flegal, 2014).

Además de la edad de los sujetos, sería también aconsejable plantear futuros proyectos de investigación relacionados con el HIIT en los que se tengan en cuenta las diferencias en cuanto al Índice de Masa Corporal (IMC) y el nivel de actividad física de los sujetos con DMT2, ya que los efectos del HIIT sobre la salud de estas personas podrían variar en función de dichas características individuales. En concreto, los estudios de esta revisión narrativa incluyen a sujetos sedentarios y con sobrepeso/obesidad; sólo hay un estudio (Mandrup et al., 2017) en el que los sujetos tienen un IMC clasificado como normopeso. Además, hay un estudio (Francois et al., 2016) que sí divide a los sujetos entre físicamente activos y no activos, aunque ambos son grupos normoglucémicos y, además, no se tiene en cuenta el IMC en los diferentes grupos. Por tanto, no hay ningún estudio en el que se haya dividido a los sujetos por grupos en función de sus características físicas para observar posibles diferencias, ya que sólo se ha tenido en cuenta si padecen o no DMT2 o prediabetes.

En esta misma línea, cabe mencionar que en ninguno de los estudios se encontraron diferencias significativas entre hombres y mujeres con DMT2 que realizaron un programa de entrenamiento basado en el HIIT. Aunque, al igual que ocurre con la diferencia de edad, el IMC y el nivel de actividad física de los sujetos, en los próximos años sería interesante estudiar más profundamente las posibles diferencias entre ambos sexos bajo condiciones de entrenamiento similares.

Por último, cabe subrayar que en ninguno de los estudios se evidenció o especificó ningún problema metabólico, hipoglucemia o náuseas entre los participantes debido a la intensidad tan alta de la carga. Por tanto, este tipo de participantes pueden realizar este tipo de entrenamientos intensos sin ningún problema o limitación importante.

Por lo tanto, el HIIT podría ser una estrategia alternativa de salud para las personas con DMT2 que afirman no disponer de tiempo suficiente para hacer ejercicio, ya que los beneficios en cuanto a la resistencia a la insulina y el $\mathrm{VO}_{2} \max$ son similares e incluso superiores al entrenamiento continuo, conllevando mucho menos tiempo al día y mayor adherencia (Jelleyman et al., 2015). No obstante, hay que tener en cuenta que enfoque contra las patologías metabólicas debe ser integral, por lo que se debe abordar la enfermedad utilizando entrenamientos combinados (Zanuso et al., 2017).

En resumen, esta revisión narrativa ofrece al lector algunas novedosas respuestas en lo relativo a los efectos del HIIT sobre la salud de las personas con DMT2, aunque en las intervenciones futuras será necesario atender a las limitaciones anteriormente comentadas de cara a la obtención de unos resultados más fiables y con mayor transferencia a la vida real de los pacientes. 


\section{Conclusiones}

El HIIT tiene efectos positivos en la salud de las personas con DMT2, sobre todo en lo relativo a la mejora de la condición física, la salud cardio-metabólica (control glucémico, HbA1c, resistencia a la insulina, perfil lipídico, tensión arterial y función endotelial), la composición corporal y la calidad de vida. De hecho, estos efectos son similares e incluso más notorios que los observados tras un entrenamiento aeróbico continuo de intensidad moderada (MICT).

Por lo tanto, el HIIT puede representar una buena estrategia de entrenamiento para mejorar la salud de los sujetos con DMT2 o prediabetes y prevenir los factores de riesgo cardiovasculares y metabólicos, sobre todo para aquellas personas que no disponen de mucho tiempo durante el día para practicar actividad física, ya que se trata de una actividad de alta intensidad y con un volumen de entrenamiento bajo.

No obstante, es necesario llevar a cabo más estudios científicos de intervención en el futuro para corroborar la veracidad de estos hallazgos y para comprobar con mayor evidencia científica la eficacia de las distintas metodologías de entrenamiento HIIT, ya que la gran mayoría de los diseños utilizados en las investigaciones incluidas en esta revisión son de tipo aeróbico (sólo un estudio incluye ejercicios de fuerza) y, además, tienen una serie de limitaciones que deberían corregirse en el futuro.

\section{Referencias}

Aguilera, R. E., Russell, J. G., Soto, M. M., Villegas, B. G., Poblete, C. A., \& Ibacache,A.P. (2015). Effect of high-intensity interval training on the reduction of glycosylated hemoglobin in type-2 diabetic adult patients. Medwave, 15(2), e6079-e6079.

Alvarez, C., Ramirez-Campillo, R., Martinez-Salazar, C., Mancilla, R., FloresOpazo, M., Cano-Montoya, J., \& Ciolac, E. G. (2016). Low-volume highintensity interval training as a therapy for type 2 diabetes. International journal of sports medicine, 37(09), 723-729.

Baños, R. F.(2016). Prescripción del ejercicio físico en sujetos con diabetes mellitus tipo 2 y diabetes gestacional. Retos: nuevas tendencias en educación física, deportey recreación, (29), 134-139.

Buchheit, M., \& Laursen, P. B. (2013). High-intensity interval training, solutions to the programming puzzle. Sports medicine, 43(10), 927-954.

Dehghan, M., Akhtar-Danesh, N., \& Merchant, A. T. (2005). Childhood obesity, prevalence and prevention. Nutrition journal, 4(1), 24.

Esposito, K., Maiorino, M. I., Bellastella, G., Chiodini, P., Panagiotakos, D., \& Giugliano, D. (2015). A journey into a Mediterranean diet and type 2 diabetes: a systematic review with meta-analyses. BMJ open, 5(8), e008222.

Evert, A. B., Boucher, J. L., Cypress, M., Dunbar, S. A., Franz, M. J., MayerDavis, E. J., ... \& Yancy, W. S. (2014). Nutrition therapy recommendations for the management of adults with diabetes. Diabetes care, 37(Supplement 1), S120-S143.

Fex, A., Leduc-Gaudet, J. P., Filion, M. E., Karelis, A. D., \& Aubertin-Leheudre, M. (2015). Effect of elliptical high intensity interval training on metabolic risk factor in pre-and type 2 diabetes patients: A pilot study. Journal of Physical Activity and Health, 12(7), 942-946.

Francois, M. E., Durrer, C., Pistawka, K. J., Halperin, F. A., \& Little, J. P. (2016) Resistance-based interval exercise acutely improves endothelial function in type 2 diabetes. American Journal of Physiology-Heart and Circulatory Physiology, 311(5), H1258-H1267.

Geloneze, B., Repetto, E. M., Geloneze, S. R., Tambascia, M. A., \& Ermetice, M. N.(2006). The threshold value for insulin resistance(HOMA-IR)in an admixtured population. Diabetes Research and Clinical Practice, 2(72), 219-220.

Gillen, J. B., Little, J. P., Punthakee, Z., Tarnopolsky, M. A., Riddell, M. C., \& Gibala, M.J.(2012).Acutehighintensity interval exercisereducesthepostprandial glucose response and prevalence of hyperglycaemia in patients with type 2 diabetes. Diabetes, Obesity and Metabolism, 14(6), 575-577.

Honkala, S. M., Motiani, K. K., Eskelinen, J. J., Savolainen, A., Saunavaara, V., Virtanen, K. A., ... \& Hannukainen, J. C. (2017). Exercise training reduces intrathoracic fat regardless of defective glucose tolerance. Medicine and science in sports and exercise, 49(7), 1313.

Inzucchi, S. E., \& Sherwin, R. S. (2011). Type 2 diabetes mellitus. Cecil Medicine. 24thed. Philadelphia, Pa: Saunders Elsevier.

Jelleyman, C., Yates, T., O’Donovan, G., Gray, L. J., King, J. A., Khunti, K., \& Davies, M. J. (2015). The effects of high intensity interval training on glucose regulation and insulin resistance: a meta analysis. Obesity reviews, 16(11), 942-961.

Kumar, P. R., Bhansali, A., Ravikiran, M., Bhansali, S., Dutta, P., Thakur, J. S., ... \& Walia, R. (2010). Utility of glycated hemoglobin in diagnosing type 2 diabetes mellitus: a community-based study. The Journal of Clinical Endocrinology \& Metabolism, 95(6), 2832-2835.

Little, J. P., Gillen, J. B., Percival, M. E., Safdar, A., Tarnopolsky, M. A., Punthakee, Z., ... \& Gibala, M. J. (2011). Low-volumehigh-intensity interval training reduces hyperglycemia and increases muscle mitochondrial capacity in patients with type 2 diabetes. Journal of applied physiology, 111(6), 15541560.

Chicharro, J. L., \& Mojares, L. M. L. (2008). Fisiología clínica del ejercicio. Ed. Médica Panamericana.

Madsen, S. M., Thorup, A. C., Bjerre, M., \& Jeppesen, P. B. (2015). Does 8 weeks of strenuous bicycle exercise improve diabetes-related inflammatory cytokines and free fatty acids in type 2 diabetes patients and individuals at high-risk of metabolic syndrome?. Archives of physiology and biochemistry, 121(4), 129138.

Madsen, S. M., Thorup, A. C., Overgaard, K., Bjerre, M., \& Jeppesen, P. B. (2015). Functional and structural vascular adaptations following 8 weeks of low volume high intensity interval training in lower leg of type 2 diabetes patients and individuals at high risk of metabolic syndrome. Archives of physiology and biochemistry, 121(5), 178-186.

Madsen, S. M., Thorup, A. C., Overgaard, K., \& Jeppesen, P. B. (2015). High intensity interval training improves glycaemic control and pancreatic â cell function of type 2 diabetes patients. PLoS One, 10(8), e0133286.

Maillard, F., Rousset, S., Pereira, B., Traore, A., Del Amaze, P. D. P., Boirie, Y., ... \& Boisseau, N. (2016). High-intensity interval training reduces abdominal fat mass in postmenopausal women with type 2 diabetes. Diabetes \& metabolism, 42(6), 433-441.

Mandrup, C. M., Egelund, J., Nyberg, M., Slingsby, M. H. L., Andersen, C. B., Løgstrup, S., ... \& Hellsten, Y. (2017). Effects of high-intensity training on cardiovascular risk factors in premenopausal and postmenopausal women. American journal of obstetrics and gynecology, 216(4), 384-e1.

Mangiamarchi, P., Caniuqueo, A., Ramirez-Campillo, R., Cardenas, P., Morales, S., Cano-Montoya, J., ... \& Alvarez, C. (2017). Effects of high-intensity interval training and nutritional education in patients with type 2 diabetes. Revista medica de Chile, 145(7), 845-853.

Ochoa-Martínez, P. Y., Hall-López, J. A., Diaz, D. P., Trujillo, D. A. Z., \& Teixeira, A. M. (2019). Effects of three months of water-based exercise training on metabolic syndrome components in older women. Retos: nuevas tendencias en educación física, deporte y recreación, (35), 181-184.

Ogden, C. L., Carroll, M. D., Kit, B. K., \& Flegal, K. M. (2014). Prevalence of childhood and adult obesity in the United States, 2011-2012. Jama, 311(8), 806-814.

Phillips, B. E., Kelly, B. M., Lilja, M., Ponce-González, J. G., Brogan, R. J., Morris, D. L., ... \& Rooyackers, O. (2017). A practical and time-efficient highintensity interval training program modifies cardio-metabolic risk factors in adults with risk factors for type II diabetes. Frontiers in endocrinology, 8, 229.

Poblete Aro, C. E., Guzmán, R., Antonio, J., Soto Muñoz, M. E., \& Villegas González,B.E.(2015). Effects of high intensity interval training versus moderate intensity continuous training on the reduction of oxidative stress in type 2 diabetic adult patients: CAT. Medwave, 15(07).

Salas-Salvadó, J., Bulló, M., Estruch, R., Ros, E., Covas, M. I., Ibarrola-Jurado, N., ... \& Romaguera, D. (2014). Prevention of diabetes with Mediterranean diets: a subgroup analysis of a randomized trial. Annals of internal medicine, 160(1), 1-10.

Støa, E. M., Meling, S., Nyhus, L. K., Strømstad, G., Mangerud, K. M., Helgerud, J., ... \& Støren, Ø. (2017). High-intensity aerobic interval training improves aerobic fitness and HbA1c among persons diagnosed with type 2 diabetes. European journal of applied physiology, 117(3), 455-467.

Umpierre, D., Ribeiro, P.A., Kramer, C. K., Leitão, C. B., Zucatti, A. T., Azevedo, M. J., ... \& Schaan, B. D. (2011). Physical activity advice only or structured exercise training and association with HbA1c levels in type 2 diabetes: a systematic review and meta-analysis. Jama, 305(17), 1790-1799.

Zanuso, S., Sacchetti, M., Sundberg, C. J., Orlando, G., Benvenuti, P., \& Balducci, S. (2017). Exercise in type 2 diabetes: genetic, metabolic and neuromuscular adaptations. A review of the evidence. Br J Sports Med, 51(21), 1533-1538. 